Daniel Weidner

\title{
Berufung, Erweckung und lebendige Lehrart. Johann Gottlieb Fichtes Reden über Wissenschaft
}

Im Sommersemester 1794 hält der frisch an die Universität Jena berufene Professor Johann Gottlieb Fichte eine öffentliche Vorlesung, die als De officiis Eruditorum Moral für Gelehrte im Vorlesungsverzeichnis angekündigt wird. Sie erweist sich als großer Erfolg: Während Fichtes Privatvorlesung über Wissenschaftslehre nur mäßig besucht ist, zieht die Moral für Gelehrte zahlreiche Hörer an, wie Fichte in einem Brief an seine Frau zufrieden feststellt: „Die Laufbahn ist gut eröffnet.“

\footnotetext{
Meine öffentlichen Vorlesungen halte ich in dem grösten Auditorium, das es in Jena giebt, und dennoch stehen noch immer eine Menge Menschen vor der Thüre; gestern Abend hat mir die halbe Universität eine sehr sollenne Musik, u. ein Vivat gebracht; u. es ist sehr glaublich, daß ich gegenwärtig wohl der geliebteste unter allen hiesigen Profeßoren bin [...]. Mithin werden meine Privat-Vorlesungen ins künftige auch sehr stark besetzt seyn. ${ }^{1}$
}

Die ersten vier seiner Vorträge veröffentlicht Fichte im Herbst desselben Jahres unter dem Titel Einige Vorlesungen über die Bestimmung des Gelehrten, unter anderem, um Gerüchten entgegenzutreten, er verbreite in seinen Vorlesungen revolutionäre Propaganda; wie er im Vorbericht betont, erscheint der Text „gerade so, wie er sie gehalten, ohne daran ein Wort $\mathrm{zu}$ ändern“. ${ }^{2}$ Er entwickelt zunächst die Bestimmung des Menschen als solchen, dann seine Bestimmung in der Gesellschaft, bespricht die Verschiedenheit der Stände, um schließlich in der vierten Vorlesung den Stand des Gelehrten zu thematisieren, den man eben aus seinem Bezug zur Gesellschaft verstehen müsse:

Der Gelehrte ist ganz vorzüglich für die Gesellschaft bestimmt: er ist, insofern er Gelehrter ist, mehr als irgend ein Stand, ganz eigentlich nur durch die Gesellschaft und für die Gesellschaft da; er hat demnach ganz besonders die Pflicht, die gesellschaftlichen Talente, Empfänglichkeit und Mittheilungsfertigkeit, vorzüglich und in dem höchstmöglichen Grade in sich auszubilden. ${ }^{3}$

1 Fichte 1970e: 134.

2 Fichte 1966a: 25. Vgl. zum Kontext der Vorlesungen Oesterreich \& Traub 2006: 20 ff.; Ries 2007: 126ff.; Schippan 2017: 154 ff.

3 Fichte 1966a: 55.

¿ Open Access. () 2019 Daniel Weidner, publiziert von De Gruyter. (cc)BY-NC-ND Dieses Werk ist lizenziert unter der Creative Commons Attribution-NonCommercial-NoDerivatives 4.0 Lizenz.

https://doi.org/10.1515/9783110676631-005 
Der Gelehrte hat also, modern gesprochen, eine gesellschaftliche ,Funktion“, er ist „Lehrer des Menschengeschlechtes“, der das Wissen weitergibt, und zugleich „Erzieher der Menschheit“, die in beständigem Fortschritt begriffen sei: „Er sieht nicht bloß das Gegenwärtige, er sieht auch das Künftige; er sieht nicht blos den jetzigen Standpunkt, er sieht auch, wohin das Menschengeschlecht nunmehr schreiten muß“. ${ }^{4}$ Der Fortschritt der Wissenschaft und derjenige des Menschengeschlechts stehen also in enger Korrelation, die entscheidend für die Bestimmung des Gelehrten ist:

Es ist ein stärkender seelenerhebender Gedanke, den jeder unter Ihnen haben kann, welcher seiner Bestimmung werth ist: auch mir an meinem Theile ist die Kultur meines Zeitalters und der folgenden Zeitalter anvertraut [...]. Ich bin dazu berufen, der Wahrheit Zeugniß zu geben; an meinem Leben, und an meinen Schicksalen liegt nichts; an den Wirkungen meines Lebens liegt unendlich viel. Ich bin ein Priester der Wahrheit; ich bin in ihrem Solde; ich habe mich verbindlich gemacht, alles für sie zu thun und zu wagen, und zu leiden. ${ }^{5}$

Die Topoi, die hier aufgerufen werden, sind alle schon bekannt und unterscheiden sich nicht grundsätzlich von denen anderer akademischer Einführungsreden, wie sie bereits in der humanistischen Universität Tradition haben, allenfalls ist das Pathos des Zeugen der Wahrheit noch gesteigert. Doch das ist nicht zufällig der Fall: Denn die Assoziation der Wissenschaft mit dem Fortschritt, die zentral für Fichtes Idee der Wissenschaft und überhaupt für ein modernes, forschungsorientiertes Wissenschaftsverständnis ist, stellt das Subjekt der Wissenschaft vor ein Problem. Dass „an meinem Leben und an meinen Schicksalen nichts liegt“, ist ja nicht nur eine Anspielung auf eine mögliche Verfolgung des Wissenschaftlers - Fichte sollte das selber wenig später im Zusammenhang mit dem Atheismusstreit erleben ${ }^{6}$-, es liegt auch in der Eigenlogik der so gedachten Wissenschaft, dass ihre Resultate von vornherein dazu bestimmt sind, überholt zu werden, dass das Wissen, das ich erworben habe, nicht mehr ein Schatz von Gelehrsamkeit ist, den man anhäufen und besitzen kann, sondern immer nur ein Durchgangsstadium, wie Fichte schon am Schluss seines Aufsatzes Über den Begriff der Wissenschaftslehre ausgeführt hatte: „Jeder Zuwachs, den die Wissenschaft erhält, vermehrt die Pflichten ihrer Diener“. ${ }^{7}$ Gerade deshalb bedarf der Gelehrte auch einer besonderen „Moral“, muss um

4 Ebd.: $56 f$.

5 Ebd.: 58.

6 Zum Atheismusstreit vgl. Ries 2007: 139-154, zu dessen Auswirkungen auf Fichte vgl. auch Oesterreich \& Traub 2006: $142 \mathrm{ff}$.

7 Fichte 1965: 153. 
seine ,Berufung' wissen und muss über die Gegenwart hinweg in die Zukunft schauen.

Die zukunftsorientierte Wissenschaft stellt aber nicht nur moralische, sondern auch epistemologische Probleme, oder genauer: ihr moralisches Problem hat epistemologische Konsequenzen. Denn selbst wenn vorausgesetzt wird, dass Wissenschaft sich immer voraus ist, dass gemäß dem Forschungsimperativ jedes Wissen auf seine eigene Überwindung drängt, so gilt das zunächst nur innerhalb der Wissenschaft. Wie ist es aber möglich, dieses Wissen auch außerhalb der Wissenschaft zu kommunizieren, und wie ist es möglich, von außen in die Wissenschaft einzutreten? Denn offensichtlich kann es für eine wesentlich von der Zukunft her gedachte und in stetigem Wandel begriffene Wissenschaft nicht einfach eine Propädeutik geben, die gewissermaßen das Resultat des Wissens im Umriss zeichnet. Die Einführung muss anders vor sich gehen, sie kann nicht einfach selbständig sein, sich aber auch noch nicht als Wissenschaft voraussetzen - wie denn der Ausdruck ,Wissenschaft‘ in Über die Bestimmungen der Gelehrten auch verhältnismäßig selten ist. Möglich ist der Vorgriff auf die Wissenschaft nur durch ein Zusammenspiel von Gattungen, denn wie der anfangs zitierte Brief zeigt, betrachtet Fichte die Wissenschaftslehre und die öffentlichen Vorlesungen als zwei parallele Projekte, als zwei Seiten derselben Sache: Die öffentlichen Vorlesungen richten sich allgemein an Jünglinge und eben an zukünftige Gelehrte, die später, konkret im nächsten Semester, überhaupt erst verstehen können, was Wissenschaft ist, wenn sie die Wissenschaftslehre besuchen. Das Wissen hat hier gewissermaßen eine Außen- und eine Innenseite, eine öffentliche und eine private oder eine exoterische und eine esoterische Dimension.

Dieses Nebeneinander und doch Ineinander zweier Vortragsweisen, so die These der folgenden Ausführungen, ist charakteristisch für die deutsche Universität des langen 19. Jahrhunderts, in der in zwei formal, institutionell und auch medial markant unterschiedenen Formen gesprochen wird: einerseits im mündlichen Vortrag vor Anfängern und andererseits im privateren Seminar oder in den jeweiligen Fachpublikationen. Es gibt zwei Adressierungen, die sich im Idealfall wechselseitig unterstützen, so dass die wissenschaftliche Autorität in öffentliche konvertiert werden kann und vice versa, deren Differenz aber auch einen weiten Spielraum, ja eine Notwendigkeit für verschiedene Rhetoriken der Wissenschaft lässt, die von entscheidender Bedeutung für die Entwicklung, das Selbstbild und die gesellschaftliche Verortung dieser neuen Wissenschaft sind. Im Raum dieser beiden Modi entfaltet sich eine Rede über Wissenschaft, die sich weniger in bestimmten Terminologien, Nomenklaturen oder sprachlichen Konventionen manifestiert als in einem rhetorischen und pragmatischen Modus des Sprechens, der für die Wissenschaftssprache umso wichtiger ist, als dieser gewissermaßen ,unbegriffliche‘ Teil wissenschaftlichen 
Sprechens gerade dessen Vorverständnis von Wissenschaft bestimmt: Was die Begriffe selbst bereits voraussetzen, kann nicht mehr begrifflich artikuliert werden, sondern wird durch Metaphern oder rhetorische Operationen umschrieben, zu denen auch das Zusammenspiel verschiedener Vortragsweisen gehört. ${ }^{8}$ Dabei ist die Rede über Wissenschaft immer in einer gewissermaßen unbestimmten Randposition situiert, weil konstitutiv offen bleibt, ob sie selbst wissenschaftlich ist oder nicht, ob sie die Wissenschaft von ,innen“ oder ,außen“ (oder gar von ,oben' oder ,unten') beschreibt.

Im Folgenden soll zunächst kurz Fichtes Verhältnis zur Kant'schen Philosophie beleuchtet werden (1.), um dann Fichtes Programm einer ,Verlebendigung، der Philosophie auf rhetorischer Grundlage (2.) sowie seine öffentliche Anwendung in den Reden an die deutsche Nation (3.) zu untersuchen. Denn wie schon die Vorlesung Über die Bestimmung des Gelehrten zeigt, kommt die spezifische Rhetorik und Politik der Wissenschaft in der öffentlichen und propädeutischen Rede besonders deutlich zum Ausdruck.

\section{1}

Fichtes Vorlesungen verstehen sich auch als Reaktion auf ein Dilemma der eigenen Wissenschaftslehre, das zugleich charakteristisch ist für die Neuerfindung der Universität, die um 1800 in Jena beginnt. Die Würde der Forschungen an dieser Universität, so werden die zahlreichen Programmschriften betonen, liegt in ihrem programmatisch ,wissenschaftlichen' Charakter, der sie von der bloßen ,Gelehrsamkeit‘ der alten Universität unterscheide. Ihre Wissenschaftlichkeit wiederum liege in ihrem Zusammenhang mit der Philosophie, die durch die Kant'sche Transzendentalphilosophie neu begründet worden sei, und zwar eben als transzendentalphilosophische Reflexion der Bedingungen der Möglichkeit von Wissenschaft, oder in Fichtes Worten: als ,Wissenschaftslehre'. Wissenschaft ist also dort, wo ein (philosophisches) Reden über Wissenschaft möglich ist - und dieses Reden hat eine strukturelle Funktion für die Universität.

Allerdings erweist sich diese Funktion sofort als problematisch, wenn es um ihre konkrete, und das heißt auch: sprachliche Realisierung geht. Kant hatte sich dezidiert gegen eine Esoterik der Philosophie gewandt und den „Schulbegriff“ der Philosophie als eines isolierten Diskurses von deren „Weltbegriff“ als Anleiterin des Wissens unterschieden. ${ }^{9}$ Der Anspruch allgemeiner Mitteilung wurde aber von

8 Zur ,Unbegrifflichkeit‘ vgl. Blumenberg 1979: $87 \mathrm{ff}$.

9 Kant 1958: $444 \mathrm{ff}$. 
vornherein durch die Unverständlichkeit der kritischen Philosophie konterkariert, die von vielen Zeitgenossen beklagt wurde und die auch Kant selbst zugab, als er in der Vorrede zur zweiten Auflage der Kritik der reinen Vernunft einräumte, es sei ihm nicht gelungen, verständlich $\mathrm{zu}$ schreiben. ${ }^{10}$ Um dieser Unverständlichkeit Abhilfe zu schaffen, verfasst er in den 1780er und 1790er Jahren eine Reihe von exoterischen Schriften, etwa die geschichtsphilosophischen Aufsätze in der Berlinischen Monatsschrift (Idee zu einer allgemeinen Geschichte in weltbürgerlicher Absicht, Beantwortung der Frage: Was ist Aufklärung?) sowie später insbesondere Der Streit der Fakultäten. All diese Texte kreisen um den „öffentliche[n] Gebrauch seiner [eigenen] Vernunft“, nämlich „denjenigen, den jemand als Gelehrter von ihr vor dem ganzen Publikum der Lesewelt macht“; ${ }^{11}$ sie versuchen also, eine im eigentlichen Sinne populäre Philosophie zu entwickeln. Aber sie wenden sich nicht nur an die Öffentlichkeit, sondern denken auch über diese nach, denn in diesen Texten entfaltet Kant auch seine politische Philosophie. Die Frage nach der Mitteilbarkeit des Wissens, die rein philosophisch keine befriedigende Antwort fand, lässt sich für Kant nur durch den Umweg eines Nachdenkens über die Orte und Institutionen einer solchen Mitteilung beantworten: über die Öffentlichkeit und - in der Fakultätsschrift über die Universität. Damit die Philosophie zur Welt kommt, muss sie über die Orte nachdenken, an denen sie die Welt berührt, genauer, sie muss die Orte so bestimmen, dass ihr der Kontakt zur Welt möglich wird, sie aber zugleich vor ihr geschützt wird. Denn ein solches Nachdenken impliziert auch eine Auseinandersetzung mit dem absolutistischen Staat einerseits, der als treibende Kraft hinter der neuen Universität steht, und mit der Theologie andererseits, die traditionell als ,höchste“ Wissenschaft auch den Führungsanspruch innerhalb der Universität beansprucht.

Kant ist dabei durchaus vorsichtig und spielt den Staat gegen die Theologie aus und umgekehrt. ${ }^{12}$ Denn er denkt die Öffentlichkeit nicht schrankenlos, sondern hält an der Unterscheidung von öffentlichem und gelehrtem Vernunftgebrauch fest, die insbesondere sein Konzept der Universität prägt. In Der Streit der Fakultäten wird das Verhältnis zwischen Staat und Vernunft, zwischen Macht und Wahrheit durch eine Art institutionelle Topologie stabilisiert, in der die oberen Fakultäten - Medizin, Theologie, Jurisprudenz - unmittelbar mit der Welt und damit auch mit staatlicher Autorität zu tun haben, während die Philosophische Fakultät sich zunächst an die inneruniversitäre Öffentlichkeit

10 Vgl. dazu Thouard 2016.

11 Kant 1964a: 55.

12 Vgl. dazu Weidner 2007. 
richtet. ${ }^{13}$ Weil dieser gelehrte Vernunftgebrauch den Staat gar nicht unmittelbar betreffe, sei der „Streit der Fakultäten“ frei von staatlichem Einfluss zu halten; umgekehrt dürfe sich freilich auch die Philosophie nicht unmittelbar an die allgemeine Öffentlichkeit wenden, wie das die Theologie von der Kanzel aus tue: ,[W]enn der Streit vor dem bürgerlichen gemeinen Wesen (öffentlich z. B. auf Kanzeln) geführt würde [...], so wird er unbefugterweise für den Richterstuhl des Volks (dem in Sachen der Gelehrsamkeit gar kein Urteil zusteht) gezogen und hört auf, ein gelehrter Streit zu sein“ “. ${ }^{14}$ Die Unverständlichkeit der Philosophie hat hier also auch eine politische Funktion, denn sie erlaubt es, die Philosophie von staatlicher Aufsicht freizustellen und ihr damit zugleich die Rolle der Richterin im innerakademischen Streit zu geben.

Allerdings ist dieses Modell nur scheinbar stabil. Denn Kant lässt durchblicken, dass auch der öffentliche Vernunftgebrauch und insbesondere die Aufgabe der Theologie, nämlich die Erziehung und Belehrung des Volkes, auf lange Sicht bei der Philosophie in besseren Händen wäre, weil eine philosophische Vernunftreligion den Zwecken des Staates besser diene als die ewig zerstrittenen Kirchen. Signifikanterweise bedient er sich dabei selbst einer religiösen Analogie: „Auf diese Weise könnte es wohl dereinst dahin kommen, daß die Letzten die Ersten (die untere Fakultät die obere) würden, zwar nicht in der Machthabung, aber doch in Beratung des Machthabenden (der Regierung)“. ${ }^{15}$ Hier wird indirekt die gerade etablierte Hierarchie wieder infrage gestellt, indem die Philosophie sich am inneruniversitären Forum vorbei an die staatlichen Autortäten wendet und diesen ausgerechnet mit einer Anspielung auf Matthäus 19,30 suggeriert, nützlicher zu sein als die Theologie - jedenfalls in Zukunft. Im Kant'schen Modell der Universität herrscht daher eine permanente Spannung nicht nur zwischen der öffentlichen und der gelehrten und der offenen und verdeckten Rede, sondern auch zwischen der Gegenwart und der Zukunft. Mithilfe der religiösen Metaphorik kann der philosophische Diskurs die Theologie beerben und unter der Hand seinen eigenen Führungsanspruch sowohl innerhalb der Universität als auch über ihre Grenzen hinweg für eine zukünftige Öffentlichkeit etablieren. ${ }^{16}$ Denn der theologische Diskurs des Übernatürlichen und Übervernünftigen, der mit der Kant'schen Kritik der

13 Zum Kontext der Fakultätsschrift vgl. Howard 2006: 121ff. Howard betont auch die Bedeutung pädagogischer Überlegungen zum Konzept der Universität, vgl. ebd.: 138 ff.

14 Kant 1964b: 298.

15 Ebd.: 299f.

16 Nicht zufällig hatte Kant auch die Öffentlichkeit mit der religiösen Figur der unsichtbaren Kirche figuriert. Ähnlich wie in den zeitgenössischen kunstreligiösen Diskursen erlaubt auch hier der Rückgriff auf religiöse Rede, die nun ,Autonomie‘ beanspruchenden neuen Diskurse zu situieren, gerade weil diese Rede selbst fluide geworden ist. 
rationalen Theologie seinen epistemischen Ort verloren hat, verfügt immer noch über Metaphern und Denkfiguren, die es möglich machen, auch über die Grenzen der neu gefassten Wissenschaft zu sprechen.

\section{2}

Für Fichte wird die Darstellungsproblematik zum eigentlichen Anstoß des Philosophierens, die eigene Philosophie versteht er lange nur als verbesserte Darstellung der Kant'schen Lehre. Dabei geht es nicht nur um die argumentative Abfolge, vor allem die Anwendung des deduktiven Prinzips, etwa dort, wo er Kants Kritik der reinen Vernunft vorwirft, sie enthalte zu viele Abschweifungen und müsse verdichtet werden. Ebenso geht es nicht ausschließlich um die Einführung einer neuen Begrifflichkeit der Philosophie - bei Fichte etwa der emphatische Begriff des ,Ich' und eben auch derjenige der ,Wissenschaft‘. Beide Bemühungen sind einem umfassenderen Bestreben untergeordnet, die sprachliche Performanz der Philosophie überhaupt zu erneuern - am deutlichsten im Medienwechsel zu einer gesprochenen Philosophie. Denn wo Kant - gerade in seinen exoterischen Schriften - ganz auf den gelehrten Schriftsteller setzt, zieht Fichte die lebendige Rede der toten Schrift vor, weil nur jene es erlaube, das Gedachte immer wieder neu und aktuell entstehen zu lassen. „Der Buchstabe tödtet ganz besonders in der Wissenschaftslehre. “17

Diese ,Verlebendigung، des Denkens hat verschiedene Quellen und verschiedene Folgen. Erstens hat Fichte ein deutlich positiveres Verhältnis zur Rhetorik als Kant oder die Tradition der Aufklärung insgesamt. Fichte schreibt im Rückblick, er habe sich in seiner Jugend für die Kanzel bestimmt gesehen, und trägt sich eine Weile mit dem Plan, in der Schweiz eine Rednerschule zu gründen. ${ }^{18}$ Die Rhetorik wird dabei auch epistemologisch und anthropologisch begründet: Ihre Funktion ist es, das unmittelbare Gefühl oder die Anschauung der Wahrheit auszudrücken, und dieser Ausdruck ist nicht nur in der Anwendung begründet, sondern in der vorreflexiven Natur der unmittelbaren Einsicht in das Selbstbewusstsein, das eben als Anschauung vorhanden ist, bevor es begrifflich artikuliert werden kann. Dem entspricht auch eine dezidiert instrumentalistische Auffassung der Sprache, die Fichte in einem frühen Aufsatz von 1795 (Von der Sprachfähigkeit und dem

17 Fichte 1971: 325. Vgl. dazu Villacañas 1993.

$18 \mathrm{Zu}$ Fichtes früher Beschäftigung mit der Rhetorik vgl. Schippan 2017: 165 ff. sowie Oesterreich \& Traub 2006: 70 ff.; hier wird insbesondere der „Ausfall“ der Ästhetik (ebd.: 81) in Fichtes Denken betont, deren Rolle wesentlich die Rhetorik übernimmt. 
Ursprung der Sprache) als „Ausdruck unserer Gedanken durch willkürliche Zeichen" bestimmt. ${ }^{19}$

Die Vorliebe für das lebendige Wort ist zweitens im Primat der praktischen Vernunft begründet, das Fichtes Philosophie noch stärker prägt als die Kants. Dabei wird dieses Primat bereits selbstkritisch auch gegen die Philosophie gewendet, wie etwa in einem kleinen Text von 1798, Ascetik als Anhang zur Moral, in dem Fichte nach einem „Gegenmittel gegen das Verderben der bloß spekulativen Stimmung insbesondere“ ${ }^{\text {20 }}$ sucht: Eine solche Stimmung entstehe aus dem Missbrauch der Reflexion, führe zu grenzenlosem Sichverlieren in die subjektive Selbstbetrachtung, in den unendlichen Regress - und gegen sie helfe nur das praktische Tätigsein, die Wendung nach außen. Insofern ist die exoterische Seite der Philosophie hier nicht mehr einfach ihre Anwendung auf die Wirklichkeit, sondern ein notwendiges Korrektiv der Spekulation.

Schließlich beruht Fichtes Projekt einer Verlebendigung der Philosophie drittens auf einer Kritik der literarischen Öffentlichkeit. Im Anschluss an die aufklärerische Kritik an der Lesesucht behauptet Fichte, das viele Lesen habe zur Lethargie geführt, die auch das Denken lähme, so etwa in Die Grundzüge des gegenwärtigen Zeitalters:

[D]as Zeitalter kann nicht mehr lesen, und darum ist alles Schreiben vergeblich. Dann wird es hohe Zeit, etwas neues zu beginnen. Dieses Neue ist nun meines Erachtens dies, daß man, von der Einen Seite, wiederum das Mittel der mündlichen Mittheilung ergreife, und diese zur Fertigkeit, und Kunst ausbilde; von der andern, sich Empfänglichkeit für diese Art der Mittheilung zu erwerben suche. ${ }^{21}$

Alle drei Faktoren machen Fichte äußerst sensibel für Darstellungsprobleme und tragen zu seiner Absicht bei, im Deutschen „eine philosophische Sprache zu schaffen“, also „der Philosophie auch eine geschmeidigere, u. besonders eine teutsche Mundart zu verschaffen“.22 Schon früh will Fichte, „statt die trokne Speculation fortzusetzen, Pläne zur Mittheilung derselben in meinen Vorlesungen entwerfen“. ${ }^{23}$ Die verschiedenen Fassungen der Wissenschaftslehre versuchen, immer bessere Darstellungen zu finden, da diese Lehre „auf

19 Fichte 1966b: 97. Zu Fichtes in der Rezeption weitgehend vernachlässigter Sprachauffassung vgl. auch Surber 1996.

20 Fichte 1979: 73.

21 Fichte 1991: $263 \mathrm{f}$.

22 Fichte 1970b: $47 \mathrm{f}$.

23 Fichte 1970c: 55. 
unendlich mannigfaltige Art vorzutragen“ sei. ${ }^{24}$ Sie werden allerdings zugleich vom deutlichen - und wachsenden - selbstkritischen Bewusstsein getragen, auch selbst unverständlich zu bleiben. Schon 1797 eröffnet er den Versuch einer neuen Darstellung der Wissenschaftslehre mit der Betonung, dass die Sprache hier unzureichend sei: „Die SprachZeichen nämlich sind durch die Hände der Gedankenlosigkeit gegangen, und haben etwas von der Unbestimmtheit derselben angenommen; man kann durch sie sich nicht sattsam verständigen. “25 1801, im Sonnenklaren Bericht an das größere Publikum über das Wesen der neuesten Philosophie, klagt Fichte noch ausführlicher, seine Philosophie werde missverstanden; der Grund dafür liege darin,

daß die Wissenschaftslehre mit einem Vortrage in der vorhandenen Wörtersprache anheben mußte. Hätte sie sogleich anfangen können, wie sie freilich endigen wird, dadurch, daß sie sich ein ihr durchaus eigenthümliches Zeichen-System geschaffen hätte, dessen Zeichen nur ihre Anschauungen, und die Verhältnisse derselben zu einander, und schlechthin nichts außer diesen, bedeuten, so hätte sie freilich nicht mißverstanden werden können, aber sie würde auch nie verstanden worden, und aus dem Geiste ihres ersten Urhebers in andere Geister übergegangen seyn. Jetzt aber hat sie das schwierige Unternehmen zu bestehen, von der Verworrenheit der Wörter aus [...] andere zur Anschauung zu leiten. ${ }^{26}$

Allerdings zieht Fichte aus der Missverständlichkeit der „vorhandenen Wörtersprache“ keineswegs den Schluss, man müsse eine eindeutige Terminologie entwickeln - im Gegenteil bedient sich seine Spätphilosophie vermehrt der Alltagssprache und der bildhaften Rede. ${ }^{27}$ Auch hier betont Fichte zwar immer wieder die Beschränktheit der Sprache: Der Lehrinhalt „muß eben mit Einem Schlage begriffen werden; um so weniger lassen sich über ihn Worte machen, noch durch sie dem Verständniß nachhelfen““. ${ }^{28}$ Aber dieser Begrenztheit der Sprache lässt sich eben nicht mit terminologischen Mitteln abhelfen, sondern nur durch eine andere Praxis der Sprache, und zwar eine solche, in der der Rezipient die Gedankenhandlung selbst nachvollzieht. Ein solcher Nachvollzug lässt sich aber weniger durch eine wie auch immer verbesserte systematische

24 Fichte 1972: 57. Die „Uneinigkeit“ in der Frage, ob es sich hier um sachliche Modifikationen oder ,nur‘ solche der Darstellungsfragen handele „gehört schon zur Tradition der Fichte Forschung“ (Zahn 1981: 157).

25 Fichte 1970a: 272.

26 Fichte 1988: $236 \mathrm{f}$.

$27 \mathrm{Zu}$ Fichtes „Wende“ in der „Abkehr vom systembezogenen, kontextautonomen Fachterminus und die Zuwendung zum gemeinsprachlichen kontextbedingten Wort“ vgl. Siegel 2009: 69.

28 Fichte 1985: 228. 
Darstellungsweise als durch den mündlichen Vortrag erzeugen. Das geschieht vor allem durch den sogenannten genetischen Vortragsstil, der erheblich zum Lehrerfolg der idealistischen Philosophie in Jena beigetragen hat und der auch entscheidend für das Reden über Wissenschaft ist, weil er die Spannungen und Paradoxien der neuen, forschungsorientierten Wissenschaft und ihrer Institution, der Universität, auf markante Weise sprachlich ausdrückt.

Fichtes Vorlesungen sind ein großer Erfolg und ziehen bald eine ganze Fülle von Hörern an. Dabei sind sie, wie er an Schiller schreibt, sorgefältig geplant:

Ich sezte die Popularität vorzüglich in den Gang, den ich nehme [. . .]. Nachdem die streng philosophische Disposition fertig ist, mache ich ihn nach ganz andern Grundsätzen: knüpfe an eine sehr gemeine Erfahrung an, und führe so den Faden, scheinbar nach der bloßen Ideen-Aßociation, über die aber unsichtbar das System wacht, fort, bestimme nirgends schärfer, als vor der Hand nöthig ist, bis zulezt die scharfe Bestimmung sich von selbst ergiebt. ${ }^{29}$

Der Vortrag geht also von einer philosophischen Disposition aus, nimmt dann aber einen Umweg über die Unschärfe der Ideenassoziation, um erst am Schluss wieder zu scharfer Bestimmung zu kommen. Paradoxerweise ist dabei Fichtes Vorlesungsweise zunächst weniger mündlich, da er nicht mehr wie noch Kant ein gegebenes Lehrbuch mündlich kommentiert, sondern seine Vorlesungen schriftlich ausformuliert, dabei aber bemüht ist, das Denken im Entstehen zu präsentieren:

Mein Vortrag ist immer synthetisch; ich werfe meine Gedanken nie hin, wie ich sie unsichtbar in meiner Studirstube gedacht habe, sondern ich denke sie, finde sie, entwikle sie vor den Augen der Hörer, und mit Ihnen [. . .]. Da ich fast alles wörtlich niederschreiben werde, so hoffe ich diese Art des Vortrags dann so ziemlich in der Gewalt zu haben. ${ }^{30}$

Der Vortrag wird also als mündlicher geschrieben und soll auch so rezipiert werden: Fichte insistiert darauf, dass die Schüler nicht mitschreiben, sondern allenfalls Stichworte notieren, um ohne Ablenkung seine Vorlesung mitvollziehen zu können. ${ }^{31}$ Die Stimme wird hier in der Tat zum entscheidenden Anreger des Denkvollzuges und zum Medium einer Gemeinschaft, die Fichte dann auch in anderen sozialen Formen zu instituieren sucht: in ,Conservatorien', in denen er mit den Studenten als gleichberechtigten Partnern diskutiert und die als Vorform des Seminars betrachtet werden können, in der Tischgesellschaft, an der

29 Fichte 1970 f: 339.

30 Fichte 1970d: $60 \mathrm{f}$.

31 Vgl. Fichte 1985: 25. 
sowohl andere Professoren als auch Studenten teilnehmen, und in der „Gesellschaft der freien Männer“, einer akademischen Vereinigung, in der oft Fichtes Texte verlesen und dann auch diskutiert werden. ${ }^{32}$

Fichte ist nicht der Einzige, der eine neue Form der Vorlesung praktiziert. Einige Jahre später hält Friedrich Wilhelm Joseph Schelling seine Vorlesungen Über die Methode des akademischen Studiums (1802), welche die neue Form nicht nur verwenden, sondern auch programmatisch begründen. Schelling betont dabei den philosophischen Zusammenhang allen Wissens, den es in der Mitteilung hervorzuheben gelte. Es sei

der wahre Vorzug der lebendigen Lehrart, daß der Lehrer nicht Resultate hinstellt, wie es der Schriftsteller pflegt, sondern daß er, in allen höhren Szienzen wenigstens, die Art zu ihnen zu gelangen selbst darstellt und in jedem Fall das Ganze der Wissenschaft gleichsam erst vor den Augen des Lehrlings entstehen läßt. Wie soll nun derjenige, der seine Wissenschaft nicht selbst aus eigener Konstruktion besitzt, fähig sein, sie nicht als ein Gegebenes, sondern als ein zu Erfindendes darzustellen ${ }^{33}$

Auch hier wird die Wissenschaft vor allem als Tätigkeit bestimmt, auch hier vollzieht sich die Bestimmung als Einführung in die Wissenschaft, in der die Hörer immer doppelt adressiert werden: als Noch-nicht-Philosophen und als doch Schon-mögliche-Philosophen. In einer Art „doppelten Brechung“ wird noch nicht philosophiert, aber auch nicht einfach propädeutisch von den Resultaten der Philosophie gesprochen, sondern diese Resultate entstehen im Vortrag. ${ }^{34}$ Das Problem des Anfangs der Philosophie, das für die systematischen Entwürfe des deutschen Idealismus von zentraler Bedeutung sein wird, hat hier sozusagen seine konkrete, sprachlich-didaktische Form gefunden.

Der genetische Vortragsstil trägt entscheidend dazu bei, dass die Vorlesung, die bis vor Kurzem noch als antiquierte Form der Wiederholung galt, einen festen Platz in den Entwürfen zu einer Erneuerung der Universität erhält. So betont etwa Friedrich Schleiermacher in Gelegentliche Gedanken über Universitäten im Deutschen Sinn (1808), dass alle Wissenschaft auf Mitteilungen beruhe, dass die Mitteilung alle wissenschaftlichen Tätigkeiten miteinander zu einer Einheit verbinde, und dass sich diese Einheit insbesondere im Kathedervortrag vollziehe:

32 Vgl. dazu Ries 2007: $133 \mathrm{ff}$.

33 Schelling 1990: 28.

34 Von einer „doppelten Brechung“ spricht Ziche 2008: 152. „Schelling holt die Propädeutik, die als Lehre von den Wissenschaften überhaupt in den Rubrizierungen der ALZ aus der Philosophie herausgelöst war und damit Philosophie verfachlicht und aufgewertet hatte, wieder in die Philosophie zurück, ohne damit Philosophie wieder sozusagen propädeutisieren zu wollen.“ (153) 
Zwei Elemente sind daher in dieser Art des Vortrages unentbehrlich und bilden sein eigentliches Wesen. Das eine möchte ich das populäre nennen; die Darlegung des muthmaßlichen Zustandes, in welchem sich die Zuhörer befinden, die Kunst sie auf das Dürftige in demselben hinzuweisen und auf den lezten Grund alles Nichtigen im Nichtwissen. Dies ist die wahre dialektische Kunst, und je strenger dialektisch, desto populärer. Das andere möchte ich das productive nennen. Der Lehrer muß alles was er sagt, vor den Zuhörern entstehen lassen; er muß nicht erzählen was er weiß, sondern sein eignes Erkennen, die That selbst, reproduciren, damit sie beständig nicht etwa nur Kenntnisse sammeln, sondern die Thätigkeit der Vernunft im Hervorbringen der Erkenntniß unmittelbar anschauen und anschauend nachbilden. ${ }^{35}$

Die hervorbringende, produktive Wissenschaft ebenso wie die Dialektik finden für Schleiermacher ihren angemessenen Ausdruck in der Form der Vorlesung, die eine Art Selbstgespräch des Forschers sei und daher zugleich das Hervorbringen des Wissens darstellen könne und die richtige Gesinnung der Forschung produziere:

Jede Gesinnung, die wissenschaftliche wie die religiöse, bildet und vervollkommnet sich nur im Leben, in der Gemeinschaft Mehrerer. Durch Ausströmung aus den Gebildetern, Vollkommenern, wird sie zuerst aufgeregt und aus ihrem Schlummer erwekt in den Neulingen; durch gegenseitige Mittheilung wächst sie und stärkt sich in denen die einander gleich sind. Wie nun die ganze Universität ein solches wissenschaftliches Zusammenleben ist: so sind die Vorlesungen insbesondere das Heiligthum desselben. ${ }^{36}$

Der mündliche Vortrag hat nicht nur propädeutische Bedeutung oder didaktische Funktionen, vielmehr bringt er unmittelbar eine ,Gesinnung ' hervor, die für die Wissenschaft notwendig ist. Durch den Vergleich mit der Religion erscheint die Vorlesung hier als Ritual, das alle Züge der protestantischen Wortverkündigung trägt. Die Universität wird zu einem Ort des Sprechens, freilich weniger des Dialogs unter Gleichen als des Selbstgesprächs des charismatischen Lehrers, der durch sein Wort die Gemeinde erzeugt. ${ }^{37}$ Dass diese Gemeinde - wie die protestantische Kirche - tendenziell nicht exklusiv ist, sondern auch Laien aufnimmt, unterscheidet Schleiermachers Entwurf von Fichtes Deduziertem Plan einer Universität, der zwar ebenfalls die Bedeutung des gesprochenen Wortes betont, aber an die Stelle der Vorlesung den Dialog unter Eingeweihten treten lassen will und die Universität überhaupt eher als von der bürgerlichen Welt isolierte Gegenwelt konzipiert. ${ }^{38}$ Den rhetorischen Vortrag reserviert Fichte dagegen jetzt für eine eigene Gattung, die angewandte Philosophie.

35 Schleiermacher 1998: $48 \mathrm{f}$.

36 Ebd.: 48.

37 Vgl. dazu allgemein: Clark 2006.

38 Vgl. Zöller 2008. 


\section{3}

Fichte, Schelling und Schleiermacher gehen selbstverständlich davon aus, dass der lebendige Vortrag sich auf Deutsch vollzieht. Das hat auch politische Implikationen, denn schon für Kant wurde die Universität zu dem Ort, an dem der philosophische Deutungsanspruch auf die Wirklichkeit trifft. Politisch ist die Frage der Sprache aber auch, weil die Universität nun einen nationalen Auftrag erhält: Als Einheit von Gelehrten und Laien bildet sie die Einheit der Nation ab - und zwar als sprachliche, weil die Sprache als Grund der Nation gedacht wird und dieser Grund in der Universität in Vollzug gesetzt wird bzw. sich mit dem allgemeinen Charisma einer höheren Wahrheit auflädt. Die spezifische Politik der Professoren, die hier ihren Ausgang nimmt, wird im langen 19. Jahrhundert über die liberalen Proteste etwa der Göttinger Sieben bis hin zu den nationalen Erweckungsreden der deutschen Professoren im Ersten Weltkrieg reichen. ${ }^{39}$

Der Anspruch auf geistige Führung, der in diesen Reden erhoben wird, ist freilich prekär. Denn er gründet auf der Wissenschaft, die ja unsichtbar und dem Laien unverständlich ist. Erneut spielt daher die Form der Mitteilung eine entscheidende Rolle. Nachdem Fichte im Zuge des Atheismusstreits aus Jena vertrieben worden ist, greift er erneut auf die Form der exoterischen Rede mit ihrer gesteigerten Mündlichkeit zurück und entwirft die sogenannte ,Angewandte Philosophie‘, eine Reihe von öffentlichen Vorträgen, die er in Berlin hält: Die Grundzüge des gegenwärtigen Zeitalters (1804/05), Anweisung zum seligen Leben (1806) und Reden an die deutsche Nation (1807/08). Diese in der philosophischen Rezeption oft vernachlässigten Texte sind in vieler Hinsicht stilbildend für die universitäre Rhetorik geworden und zeigen noch einmal die appellative und performative Struktur des Redens über Wissenschaft.

Der rhetorische Zweck und das rhetorische Format dieser öffentlichen Reden sind dabei von vornherein bewusst und auch explizit: Er habe sich, so Fichte in seinem Vorwort zu Die Grundzüge des gegenwärtigen Zeitalters, an ein gebildetes Publikum gewandt, daher „hielt ich sie [die Vorlesungen] in der bekannten Büchersprache, und an dem Faden der Metapher, der dieser zu Grunde liegt. Ich hätte ganz dasselbe, auch als Prediger, von der Kanzel für das Volk insbesondere vortragen können; nur hätte ich es sodann in der Bibelsprache thun müssen“. ${ }^{40}$ Die Vorlesungen folgen also dem bekannten rhetorischen Modell der Vorlesung Über die Bestimmung des Gelehrten, in dem der Gedanken-

39 Vgl. Ries 2007. Zu 1914 vgl. die klassische Studie von Lübbe 1974.

40 Fichte 1991: 276f. 
gang in eine anschauliche Sprache gekleidet wird. Dabei wird der politische Gehalt der Gedanken gerade erst in dieser Einkleidung deutlich, weil das Publikum eben kein gelehrtes, sondern ein nationales ist, und weil der Ausgangszustand, in dem es angesprochen wird, kein neutraler mehr ist, sondern einer der höchsten Entfernung von der Wahrheit:

Die Grundsätze derselben sind freilich kopfbrechende Speculationen, die keinen unmittelbaren Einfluß auf's menschliche Leben haben; aber ihre Folgen sind äußerst wichtig für ein Zeitalter, dessen Moral bis in seine Quellen verdorben ist; und diese Folgen der Welt in einem anschaulichen Lichte darzustellen, wäre, glaube ich, Verdienst um sie. ${ }^{41}$

Die Gegenwart befinde sich im „Stand der vollendeten Sündhaftigkeit“, ${ }^{42}$ Aufgabe des Redners ist es, seine Hörer aus diesem Zustand zu befreien und wieder $\mathrm{zu}$ freien Individuen zu machen. Damit wird das rhetorische Modell recht eigentlich zu einem homiletischen: Wie der protestantische Prediger spricht auch Fichte seine Hörer im Stand der „Sündhaftigkeit“ an, also als Hörer, die konstitutiv gar nicht in der Lage sind zu hören, sondern erst zu Hörern gemacht werden müssen. Die religiösen Erweckungsfiguren, die schon in der Bestimmung des Gelehrten die Zukunft der Wissenschaft vorwegnehmen sollten, sind daher hier noch stärker ausgeprägt, besonders deutlich in den Reden an die deutsche Nation.

Fichte hält die Reden an die deutsche Nation im von französischen Truppen besetzten Berlin, und bald rankt sich um sie eine reiche Mythologie - so habe er etwa gegen den Marschtritt der französischen Truppen ansprechen müssen. Zur Mythisierung und eben besonders zur religiösen Aufladung greifen aber auch die Texte selbst. Fichte ruft nun nicht mehr einzelne religiöse Metaphern wie die „Priester der Wahrheit“ auf, sondern entwickelt lang ausgesponnene religiöse Bilder. Die erste Vorlesung beschließt Fichte mit einer breit entfalteten Allegorie: Heute erscheine die Zeit wie ein Schatten, der sich über den Leichnam beugt, aber die Morgenröte sei schon angebrochen: „Ich will, so ich es kann, die Strahlen dieser Morgenröthe fassen, und sie verdichten zu einem Spiegel, in welchem die trostlose Zeit sich erblicke" ${ }^{43}$ Die dritte Vorlesung entwickelt eine lange Mahnung, ihm allen Zweifeln zum Trotz Glauben zu schenken: „Jetzt vermag es das Zeitalter noch gar nicht, unsern Worten zu glauben, und es ist nothwendig, daß ihm dieselben vorkommen, wie Mährchen.“44 Dieser Unglauben gehöre

41 Fichte 1968: 171.

42 Fichte 1991: 201.

43 Fichte 2005: 116. Vgl. allgemein zur allegorischen Übergangsrhetorik Oesterreich \& Traub 2006: 344.

44 Fichte 2005: 141. 
eben zur Natur der, vollendeten Sündhaftigkeit‘; ihm gegenüber figuriert sich Fichte als Prophet, der angesichts des ,Todes' und gegenüber ,Toten` vom ,Leben` spricht: „Höre dieses Zeitalter ein Gesicht eines alten Sehers, das auf eine wohl nicht weniger beklagenswerthe Lage berechnet war. “45 Gemeint ist damit das dann im Folgenden lang paraphrasierte 37. Kapitel des Hesekielbuches, in dem der Prophet durch das Wort des Herrn die toten Gebeine wieder lebendig macht - wie eben die gegenwärtige Rede die Hörer wieder erweckt:

\begin{abstract}
Lasset immer die Bestandtheile unsres höhern geistigen Lebens eben so ausgedorret, und eben darum auch die Bande unserer National-Einheit eben so zerrissen, und in wilder Unordnung durcheinander zerstreut herumliegen, wie die Todtengebeine des Sehers [...]; der belebende Odem der Geisterwelt hat noch nicht aufgehört zu wehen. Er wird auch unsers Nationalkörpers erstorbene Gebeine ergreifen, und sie aneinanderfügen [. . . ${ }^{46}$
\end{abstract}

Das Zusammenfügen und Wiederbeleben des Nationalkörpers wird nun - ganz wie die Prophetie selbst - wesentlich als sprachlicher Vollzug verstanden. Dabei thematisiert Fichte nicht nur sein eigenes sprachliches Handeln, sondern macht auch die Sprache selbst zum Gegenstand der Reflexion. Denn sie ist nicht nur der Anschauungskern einer Nation, die auch im Verborgenen existiert und sich durch ihre Sprache aus der Verborgenheit heraus wieder erheben kann. Auch die wichtigen Kategorien und Unterscheidungen wie die zentrale Unterscheidung von ,Leben' und ,Tod' werden als sprachliche gefasst. In der berühmten vierten Vorlesung erklärt Fichte die deutsche Sprache zu dem, was die Deutschen eigentlich zur Nation mache. Zugleich bestimmt er sie inhaltlich, und zwar durch die Unterscheidung von anderen, romanischen Sprachen. Während nämlich die romanischen Völker ihre Sprache einmal gewechselt hätten und latinisiert wurden, hätten die Deutschen ihre ursprüngliche Sprache fortgesprochen. Dieser Unterschied, wohl von August Wilhelm Schlegel übernommen, wird dann im Folgenden nicht nur zu einer so schematischen wie polemischen Theorie der Sprachnation ausgebaut, die ganz auf der Gegenüberstellung von lebendigem Deutschen und totem „Neulatein“ beruht. ${ }^{47}$ Er ist auch entscheidend für die Wirkung, die Fichtes Reden für sich imaginieren, weil er das jeweilige nationale Verhältnis zur Bildung betrifft, wie Fichte in immer neuen Anläufen ausführt:

Beim Volke der lebendigen Sprache greift die Geistesbildung ein ins Leben; beim Gegentheile geht geistige Bildung, und Leben jedes seinen Gang für sich fort. [.. .] Aus demselben Grunde ist es einem Volke der ersten Art mit aller Geistesbildung rechter eigentlicher

45 Ebd.

46 Ebd.: 142.

47 Vgl. dazu Müller-Vollmer 1981. 
Ernst, und es will, daß dieselbe ins Leben eingreife; dagegen einem von der letztern Art diese vielmehr ein genialisches Spiel ist, mit dem sie nichts weiter wollen. Die letztern haben Geist; die erstern haben zum Geiste auch noch Gemüth. ${ }^{48}$

Die politische Wirkung des eigenen Sprechens wird damit begründet, dass in der lebendigen Sprache auch die abstrakten Ausdrücke auf konkrete Bedeutungen zurückzuführen sind, während sie in der toten Sprache abgeschnitten von ihrer lebendigen Wurzel, ohne Anschauung und daher eigentlich leer seien Fichtes berühmte Beispiele sind „Humanität“, „Popularität“, „Liberalität“, die für den Deutschen als Fremdwörter nichts bedeuten und eigentlich auch für den Franzosen gelehrt abgeleitet werden müssten. Dabei sei es nicht nur und nicht einmal primär die politische Sprache, die durch die Abgrenzung vom Sprachgebrauch erstarre, sondern noch deutlicher die Sprache der Bildung. In den neulateinischen Sprachen gebe es eine Trennwand zwischen Gelehrsamkeit und Alltag, Bildung und Leben. Ihre Bildungssprache entwickele sich für sich und könne wie bei den Alten zu einem ,goldenen Zeitalter' führen, in dem das Wissen vollständig formuliert sei. Aber gerade das, so Fichte, werde ein ,geistiger Betrachter' als Erstarrung wahrnehmen:

Goldene Zeitalter in jeder Rüksicht sind ihm eine Beschränktheit der Erstorbenheit. Das Gold möge zwar das edelste seyn im Schooße der erstorbenen Erde, meint er, aber des lebendigen Geistes Stoff sey jenseit der Sonne, und jenseit aller Sonnen, und sei ihre Quelle. Ihm wikelt sich die Geschichte, und mit ihr das Menschengeschlecht, nicht ab nach dem verborgenen und wunderlichen Gesetze eines Kreistanzes, sondern nach ihm macht der eigentliche und rechte Mensch sie selbst, nicht etwa nur wiederholend das schon dagewesene, sondern in die Zeit hinein erschaffend das durchaus neue. ${ }^{49}$

Die radikale Zukünftigkeit der Wissenschaftskonzeption, die nicht auf ein festes Wissen oder einen Schatz von Gelehrsamkeit setzt, sondern auf Wissenschaftlichkeit als Prinzip der Hervorbringung des Neuen, fordert also eine Sprache in Bewegung. Fichte begründet diese Bewegung auf doppelte Weise: Die lebendige Sprache sei mit der Vergangenheit verbunden und verändere sich aus dieser heraus immer wieder neu; und sie sei die Sprache der Nation, in der die verschiedenen Stände zusammenleben und sich befruchten. Dabei ist auch jene Sprache wie diese Gemeinschaft etwas zuallererst Hervorzubringendes, und zwar durch eine Rede, deren Anschaulichkeit das gelehrte und das ungelehrte Publikum verbinden kann und die sich zugleich auch nach außen abgrenzt und politisiert, indem sie sich von dem her versteht, was sie nicht ist: hier eben das Französische. Beides, Vorgriff und Abgrenzung, ist freilich nur

48 Fichte 2005: $155 \mathrm{f}$.

49 Ebd.: 190. 
im Vollzug möglich, nur darin, dass die Sprache zum Sprechen gebracht wird, dass die Verbindung vollzogen wird, und dass auch die Adressaten in diese Verbindung einbezogen werden.

Die wesentliche Adressiertheit dieses Sprechens wird nirgendwo deutlicher als in der vierzehnten, abschließenden Rede, in der die Situation noch einmal ausgestaltet wird und die Hörer noch einmal zum Hören aufgefordert werden:

Die Reden, welche ich hierdurch beschließe, haben freilich ihre laute Stimme zunächst an Sie gerichtet, aber sie haben im Auge gehabt die ganze deutsche Nation [...]. Merket ihr jetzo nicht auf, und gehet in euch, lasset ihr auch diese Reden wieder als einen leeren Kützel der Ohren, oder als ein wunderliches Ungethüm an euch vorüber gehen, so wird kein Mensch mehr auf euch rechnen. Endlich einmal höret, endlich einmal besinnt Euch. Geht nur dieses mal nicht von der Stelle, ohne einen festen Entschluß gefaßt zu haben [. . . . ${ }^{50}$

Öffentlichkeit wird hier nicht durch deliberatives Aushandeln oder durch dialogische Kommunikation hergestellt, sondern als eine Gemeinschaft von Hörern, die freilich erst zu Hörern erweckt werden müssen, indem sie ihre Verblendung ablegen, um sich dann frei zum Handeln zu entschließen. ${ }^{51}$ Freiheit wie auch Zukunft sind dabei das Produkt des rhetorischen Appells - wie in dem Erziehungsprogramm der Vorlesung Über die Bestimmung des Gelehrten soll auch hier der Zuhörer ein Subjekt allererst werden. Wie die Wissenschaft wird auch die Nation frei und autonom, wenn sie vor die Alternative gestellt wird, was sie werden will: „Es hängt von euch ab, ob ihr das Ende seyn wollt [. . . oder ob ihr der Anfang seyn wollt und der Entwiklungspunkt einer neuen, über alle eure Vorstellungen herrlichen Zeit“. 52

\section{4}

Trotz der großen Bemühungen Fichtes, zum Verständnis seiner Philosophie anzuleiten, ist vielfach überliefert worden, wie überfordert seine Hörer waren. So etwa von Henrik Steffens:

„Meine Herren“, sprach er, „fassen Sie sich zusammen, gehen sie in sich ein, es ist hier
von keinem Äußern die Rede, sondern lediglich von uns selbst.“- Die Zuhörer scheinen
so aufgefordert, wirklich in sich zu gehen. Einige veränderten die Stellung und richteten
sich auf, andere sanken in sich zusammen und schlugen die Augen nieder; offenbar aber
erwarteten alle mit großer Spannung, was nun auf diese Aufforderung folgen sollte.

50 Ebd.: 285.

51 Zur Unterscheidung von deliberativer und prophetischer Rhetorik hilfreich: Kaveny 2016.

52 Fichte 2005: 288. 
„Meine Herren“, fuhr darauf Fichte fort, „denken Sie die Wand“, - ich sah es, die Zuhörer dachten wirklich die Wand und es schien ihnen allen zu gelingen. - „Haben Sie die Wand gedacht?“ fragte Fichte. „Nun, meine Herren, so denken Sie denjenigen, der die Wand gedacht hat.“ - Es war seltsam, wie jetzt offenbar eine Verwirrung und Verlegenheit zu entstehen schien. Viele Zuhörer schienen in der Tat denjenigen, der die Wand gedacht hatte, nirgends entdecken zu können, und ich begriff nun, wie es wohl geschehen könnte, daß junge Männer, die über den ersten Versuch zur Spekulation auf eine so bedenkliche Weise stolperten, bei ihren ferneren Bemühungen in eine sehr gefährliche Gemütsstimmung geraten konnten. ${ }^{53}$

Dieses Scheitern der Vermittlung der spekulativen Philosophie, die, so etwa Jean Paul in seiner Vorrede zur Clavis Fichteana, „mehr in den Ohren als in den Köpfen gewesen" sei, ${ }^{54}$ bezeugt freilich nicht notwendig das Scheitern des gesamten Unternehmens. Im Gegenteil wird dieses ja gerade durch die Verschränkung von Unverständlichkeit und behaupteter Evidenz, von Entrücktheit und Überlegenheitsanspruch gegenüber der Welt angetrieben. Herrschend kann diese Philosophie gerade darum werden, weil sie nicht sofort verstanden wird exoterische und esoterische Seite bedingen einander und resultieren in einer neuen Strategie der Wahrheit, die für die deutsche Universität des 19. Jahrhunderts charakteristisch bleiben wird, in der der Geist der reinen Forschung einhergeht mit dem Anspruch auf kulturelle Überlegenheit und die daher auch immer eine Fülle von Anekdoten von jenen überliefert, die diesem Anspruch nicht gerecht werden.

Für Fichte war das öffentliche Reden über Wissenschaft zunächst eine „Publikumsstrategie“ zur Herstellung einer philosophischen Öffentlichkeit für die neue Philosophie, nicht zuletzt auch in seiner Auseinandersetzung mit der Staatsmacht. ${ }^{55}$ Schnell wird diese Strategie allerdings weit mehr als ein Mittel sie wird zum essentiellen Bestandteil einer Philosophie, die sich wesentlich in die Zukunft hinein entwirft. In der Evokation einer zukünftigen Hörerschaft vollzieht sich dabei ein doppelter Anspruch: ein wissenschaftlicher, der dem zukunftsbezogenen Imperativ der Forschung folgt und dabei ein radikal neues Wissen als bereits existent vorstellt; und ein politischer, der dieses Wissen gerade in seiner Reinheit als mit dem Gemeinwesen verflochten denkt und nicht nur aus der freien Forschung auf eine freie Gesellschaft schließt, sondern auch bestimmte Herrschaftsansprüche der Philosophie transportiert: den Herrschaftsanspruch einer zukünftigen deutschen Nation über die anderen Völker ebenso wie denjenigen der Philosophie über diese Nation.

53 Steffens 1841: 79.

54 Jean Paul 1966: 1013.

55 Schippan 2017: 160. 


\section{Literatur}

Blumenberg, Hans (1979): Schiffbruch mit Zuschauer. Frankfurt a.M.: Suhrkamp.

Clark, William (2006): Academic Charisma and the Origins of the Research University. Chicago, London: University of Chicago Press.

Fichte, Johann Gottlieb (1965): Über den Begriff der Wissenschaftslehre oder der sogenannten Philosophie, als Einladungsschrift zu seinen Vorlesungen über die Wissenschaft. In: Gesamtausgabe der Bayerischen Akademie der Wissenschaften. Reihe I, Bd. 2: Werke 1793-1795. Hrsg. von Reinhard Lauth \& Hans Jacob unter Mitw. von Manfred Zahn. Stuttgart-Bad Cannstatt: Frommann-Holzboog, 91-172.

Fichte, Johann Gottlieb (1966a): Einige Vorlesungen über die Bestimmung des Gelehrten. In: Gesamtausgabe der Bayerischen Akademie der Wissenschaften. Reihe I, Bd. 3: Werke 1794-1796. Hrsg. von Reinhard Lauth \& Hans Jakob unter Mitw. von Richard Schottky. Stuttgart-Bad Cannstatt: Frommann-Holzboog, 1-74.

Fichte, Johann Gottlieb (1966b): Von der Sprachfähigkeit und dem Ursprung der Sprache. In: Gesamtausgabe der Bayerischen Akademie der Wissenschaften. Reihe I, Bd. 3: Werke 1794-1796. Hrsg. von Reinhard Lauth \& Hans Jakob unter Mitw. von Richard Schottky. Stuttgart-Bad Cannstatt: Frommann-Holzboog, 91-128.

Fichte, Johann Gottlieb (1968): Fichte an Marie Johanne Rahn am 5. 9.1790. In: Gesamtausgabe der Bayerischen Akademie der Wissenschaften. Reihe III, Bd. 1: Briefwechsel 1775-1793. Hrsg. von Hans Jacob \& Reinhard Lauth. Stuttgart-Bad Cannstatt: Frommann-Holzboog, 169-174.

Fichte, Johann Gottlieb (1970a): Versuch einer neuen Darstellung der Wissenschaftslehre. In: Gesamtausgabe der Bayerischen Akademie der Wissenschaften. Reihe I, Bd. 4: Werke 1797-1798. Hrsg. von Reinhart Lauth \& Hans Gliwitzky unter Mitw. von Richard Schottky. Stuttgart-Bad Cannstatt: Frommann-Holzboog, 167-282.

Fichte, Johann Gottlieb (1970b): Fichte an Anna Henriette Schütz am 15. 1.1794. In: Gesamtausgabe der Bayerischen Akademie der Wissenschaften. Reihe III, Bd. 2: Briefwechsel 1793-1795. Hrsg. von Hans Jacob \& Reinhard Lauth. Stuttgart-Bad Cannstatt: Frommann-Holzboog, 49-51.

Fichte, Johann Gottlieb (1970c): Fichte an Karl August Böttiger am 4. 2.1794. In: Gesamtausgabe der Bayerischen Akademie der Wissenschaften. Reihe III, Bd. 2: Briefwechsel 1793-1795. Hrsg. von Hans Jacob \& Reinhard Lauth. Stuttgart-Bad Cannstatt: Frommann-Holzboog, 54-56.

Fichte, Johann Gottlieb (1970d): Fichte an Johann Kaspar Lavater im Februar 1794. In: Gesamtausgabe der Bayerischen Akademie der Wissenschaften. Reihe III, Bd. 2: Briefwechsel 1793-1795. Hrsg. von Hans Jacob \& Reinhard Lauth. Stuttgart-Bad Cannstatt: Frommann-Holzboog, 60-62.

Fichte, Johann Gottlieb (1970e): Fichte an Marie Johanne Fichte am 14.-17.6.1794. In: Gesamtausgabe der Bayerischen Akademie der Wissenschaften. Reihe III, Bd. 2: Briefwechsel 1793-1795. Hrsg. von Hans Jacob \& Reinhard Lauth. Stuttgart-Bad Cannstatt: Frommann-Holzboog, 133-137.

Fichte, Johann Gottlieb (1970 f): Fichte an Johann Christoph Friedrich Schiller am 27. 6.1795. In: Gesamtausgabe der Bayerischen Akademie der Wissenschaften. Reihe III, Bd. 2: Briefwechsel 1793-1795. Hrsg. von Hans Jacob \& Reinhard Lauth. Stuttgart-Bad Cannstatt: Frommann-Holzboog, 336-340. 
Fichte, Johann Gottlieb (1971): Ueber den Unterschied des Geistes, u. des Buchstabens in der Philosophie. In: Gesamtausgabe der Bayerischen Akademie der Wissenschaften. Reihe II, Bd. 3: Nachgelassene Schriften 1793-1795. Hrsg. von Reinhard Lauth \& Hans Jacob unter Mitw. von Hans Gliwitzky \& Peter Schneider. Stuttgart-Bad Cannstatt: FrommannHolzboog, 323-333.

Fichte, Johann Gottlieb (1972): Fichte an Karl Leonhard Reinhold am 21. 3.1797. In: Gesamtausgabe der Bayerischen Akademie der Wissenschaften. Reihe III, Bd. 3: Briefwechsel 1796-1799. Hrsg. von Reinhard Lauth \& Hans Gliwitzky unter Mitw. von Manfred Zahn \& Peter Schneider. Stuttgart-Bad Cannstatt: Frommann-Holzboog, 56-58. Fichte, Johann Gottlieb (1979): Ascetik als Anhang zur Moral. In: Gesamtausgabe der Bayerischen Akademie der Wissenschaften. Reihe II, Bd. 5: Nachgelassene Schriften 1796-1801. Stuttgart-Bad Cannstatt: Frommann-Holzboog, 55-78.

Fichte, Johann Gottlieb (1985): Die Wissenschaftslehre. In: Gesamtausgabe der Bayerischen Akademie der Wissenschaften. Reihe II, Bd .8: Nachgelassene Schriften 1804. Hrsg. von Reinhard Lauth \& Hans Gliwitzky unter Mitw. von Erich Fuchs. Stuttgart-Bad Cannstatt: Frommann-Holzboog.

Fichte, Johann Gottlieb (1988): Sonnenklarer Bericht an das größere Publikum über das eigentliche Wesen der neuesten Philosophie. In: Gesamtausgabe der Bayerischen Akademie der Wissenschaften. Reihe I, Bd. 7: Werke 1800-1801. Hrsg. von Reinhard Lauth \& Hans Gliwitzky. Unter Mitw. von Erich Fuchs \& Peter K. Schneider. Stuttgart-Bad Cannstatt: Frommann-Holzboog, 165-274.

Fichte, Johann Gottlieb (1991): Die Grundzüge des gegenwärtigen Zeitalters. In: Gesamtausgabe der Bayerischen Akademie der Wissenschaften. Reihe I, Bd. 8: Werke 1801-1806. Hrsg. von Reinhard Lauth \& Hans Gliwitzky. Unter Mitw. von Josef Beeler. Stuttgart-Bad Cannstatt: Frommann-Holzboog, 141-398.

Fichte, Johann Gottlieb (2005): Reden an die deutsche Nation. In: Gesamtausgabe der Bayerischen Akademie der Wissenschaften. Reihe I, Bd. 10: Werke 1808-1812. Hrsg. von Erich Fuchs et al. Stuttgart-Bad Cannstatt: Frommann-Holzboog, 1-454.

Howard, Thomas Albert (2006): Protestant Theology and the Making of the Modern German University. Oxford, New York: Oxford University Press.

Jean Paul (1966): Clavis Fichtiana, Vorrede. In: Sämtliche Werke. Hrsg. von Norbert Miller. Abt. 1, Bd. III. München: Hanser, 1011-1018.

Kant, Immanuel (1958): Logik. In: Theorie-Werkausgabe. Hrsg. von Wilhelm Weischedel. Bd. VI. Frankfurt a.M.: Suhrkamp, 419-584.

Kant, Immanuel (1964a): Beantwortung der Frage: Was ist Aufklärung? In: TheorieWerkausgabe. Hrsg. von Wilhelm Weischedel. Bd. 11. Frankfurt a.M.: Suhrkamp, 53-62.

Kant, Immanuel (1964b): Der Streit der Fakultäten. In: Theorie-Werkausgabe. Hrsg. von Wilhelm Weischedel. Bd. 11. Frankfurt a.M.: Suhrkamp, 265-393.

Kaveny, Cathleen (2016): Prophecy without Contempt. Religious Discourse in the Public Square. Cambridge (Mass.): Harvard University Press.

Lübbe, Hermann (1974): Die philosophischen Ideen von 1914. In: Ders., Politische Philosophie in Deutschland. München: DTV, 173-238.

Müller-Vollmer, Kurt (1981): Fichte und die romantische Sprachtheorie. In: Klaus Hammacher (Hrsg.), Der transzendentale Gedanke. Die gegenwärtige Darstellung der Philosophie Fichtes. Hamburg: Meiner, 442-459.

Oesterreich, Peter \& Hartmut Traub (2006): Der ganze Fichte. Die populäre, wissenschaftliche und metaphilosophische Erschließung der Welt. Stuttgart: Kohlhammer. 
Ries, Klaus (2007): Wort und Tat. Das politische Professorentum der Universität Jena im frühen 19. Jahrhundert. Stuttgart: Steiner.

Schelling, Friedrich W.J. (1990): Vorlesung über die Methode (Lehrart) des akademischen Studiums. Auf der Grundlage des Textes der Ausgabe von Otto Weiss mit Einleitung und Anmerkungen und einer Beilage „Schellings philosophisches Testament“ neu hrsg. von Walter E. Ehrhardt. Hamburg: Meiner.

Schippan, Martin (2017): Die Akademische Antrittsrede um 1800. Literarische Konstitution der philosophischen Öffentlichkeit. Heidelberg: Winter.

Schleiermacher, Friedrich (1998): Gelegentliche Gedanken über Universitäten in deutschem Sinn. Nebst einem Anhang über eine neu zu errichtende. In: Kritische Schleiermacher Gesamtausgabe. Abt. I., Bd. 6: Universitätsschriften. Herakleitos. Kurze Darstellung des theologischen Studiums. Hrsg. von Dirk Schmid. Berlin, New York: De Gruyter, 15-100.

Siegel, Martin (2009): Die Einforderung eines lebendigen Sprachvollzuges als Kennzeichen der späten Wissenschaftslehre Fichtes. In: Fichte Studien 32, 67-78.

Steffens, Henrik (1841): Was ich erlebte. Aus den Erinnerungen niedergeschrieben. Bd. 4. Breslau: Max.

Surber, Jere Paul (1996): Language and German Idealism. Fichte's Linguistic Philosophy. Amherst, New York: Humanities Press.

Thouard, Denis (2016): Geteilte Ideen. Philosophische Versuche, den Leser zum Verstehen zu bringen. Aus dem Französischen von Ulrich Kunzmann. Berlin: Matthes \& Seitz.

Villacañas, José L. (1993): Fichte und die charismatische Verklärung der Vernunft. In: Fichte Studien 5, 117-148.

Weidner, Daniel (2007): Kants Säkularisierung der Philosophie, die politische Theologie der bürgerlichen Gesellschaft und die Kritik der Bibel. In: Zeitschrift für Religions- und Geistesgeschichte 59 (2), 97-120.

Zahn, Manfred (1981): Fichtes Sprachproblem und die Darstellung der Wissenschaftslehre. In: Klaus Hammacher (Hrsg.), Der transzendentale Gedanke. Die gegenwärtige Darstellung der Philosophie Fichtes. Hamburg: Meiner, 155-167.

Ziche, Paul (2008): Philosophie als Propädeutik und Grundlage akademischer Wissenschaft. Schellings Vorlesungen über die Methode des akademischen Studiums im Kontext der Universität Jena um 1800. In: Istvan Fehér \& Peter Oesterreich (Hrsg.), Philosophie und Gestalt der Europäischen Universität (Schellingiana 18). Stuttgart-Bad Cannstatt: Frommann-Holzboog, 147-168.

Zöller, Günter (2008): „Veredelung des Menschengeschlechts durch wissenschaftliche Bildung“. Die Universität als Kunstschule des Wissenschaftlichen Verstandesgebrauches bei J.G. Fichte. In: Istvan Fehér \& Peter Oesterreich (Hrsg.), Philosophie und Gestalt der Europäischen Universität (Schellingiana 18). Stuttgart-Bad Cannstatt: FrommannHolzboog, 103-122. 